\title{
Chromospheric reversals in the emergence of an ephemeral region
}

\author{
A. Sainz Dalda ${ }^{1,2}$ and A. López Ariste ${ }^{3}$ \\ 1 THEMIS SL, c/ vía Láctea s/n, 38200 La Laguna, Tenerife, Spain \\ e-mail: asainz@themis.iac.es \\ 2 Instituto de Astrofísica de Canarias, c/ vía Láctea s/n, 38200 La Laguna, Tenerife, Spain \\ 3 THEMIS, CNRS UPS 853, c/ vía Láctea s/n, 38200 La Laguna, Tenerife, Spain
}

Received 25 May 2006 / Accepted 1 March 2007

\begin{abstract}
Context. The behaviour of both ephemeral regions and moving magnetic features has been often described for the photospheric layer, but not for the chromosphere. Both magnetic structures are related to the decay of active regions, but their actual role is not yet clear. Aims. Our aim is to observe and understand the behaviour of these structures in the chromosphere.

Methods. We performed simultaneous photospheric and chromospheric spectropolarimetric observations of an ephemeral region and a moving magnetic feature. A new code developed for the reduction of spectropolarimetric data of several wavelengths observed simultaneously was used here for the first time and will be described here for future reference. The resulting Stokes profiles are analysed in detail.

Results. The Stokes $V$ profiles of Fe I 6301 and $6302 \AA$ are reversed in polarity with respect to the chromospheric Ca II 8498 and $8542 \AA$ in the cases shown. They reveal a reversed magnetic field topology between these layers for both structures. The time evolution of the ephemeral region results in a cancellation of the chromospheric signal, while the ratio of Stokes $V$ amplitudes of Fe I 6301 and $6302 \AA$ simultaneously informs us of a strengthening of the photospheric field. A scenario of the evolution of the ephemeral region is consequently suggested.

Conclusions. The moving magnetic feature reveals itself as an exclusively photospheric feature, similar to the ephemeral region in the initial stages of its emergence. As the loop emerges into the chromosphere it is slowed down and does not reach the corona. Most probably, the opposite polarity background in the chromosphere prevents ascent into corona.
\end{abstract}

Key words. Sun: magnetic fields - Sun: chromosphere - techniques: polarimetric

\section{Introduction}

Ephemeral regions were first observed by Dodson (1953) and studied in detail by Harvey \& Martin (1973). Their main behavioural characteristics have been well defined by Martin (1990): an ephemeral region is a small bipolar region in which its opposite polarities i) appear almost simultaneously and very close to each other; ii) increase their magnetic flux with time; and iii) move in opposite directions as they increase in magnetic flux. A summary of their physical properties can be found in Hagenaar (2001). The mechanisms at the origin of ephemeral regions are still unknown. They appear throughout the solar photosphere roughly at a rate of $4 \times 10^{4}$ per day, contributing in several $10^{22} \mathrm{Mx} \mathrm{day}^{-1}$ to the total flux rate (see Hagenaar 2001). Despite this rate of occurrence, they contribute to the solar magnetism in ways that are not yet fully understood (Schrijver et al. 1997; Hagenaar 2001). Independently of their origin and relation with the solar dynamo, it is assumed that these bipolar regions emerge through the photosphere and into the lower corona, where they interact with the background coronal field and rearrange it, resulting occasionally in reconnections (Archontis et al. 2005). Although the fact of the emergence is obvious from the photospheric dynamics of the ephemeral regions, and also from the coronal rearrangement of field lines at their arrival, we are missing the picture of the magnetic evolution of these bipoles in their emergence process through the photospheric and chromospheric layers, where they first interact with the pervading coronal field.

We have observed an ephemeral region from its early stages of evolution and up to its arrival at the chromosphere with the MTR instrument of THEMIS. THEMIS is pretty unique in allowing full spectropolarimetry of several lines of interest simultaneously and in the absence of any parasitic instrumental polarization. We have observed photospheric and chromospheric lines simultaneously and found reversed polarities of the Stokes $V$ profiles in the chromosphere with respect to those of the photosphere (we refer to this as chromospheric reversals) in the ephemeral region, but also in a moving magnetic feature (hereafter ER and MMF respectively).

Although chromospheric reversals have often been seen to be the result of chromospheric emission processes (Sánchez Almeida 1997), this is not the case in the observations we present here. Chromospheric reversals are observationally due to a change in polarity of the magnetic field along the approximately $1000 \mathrm{~km}$ separating the formation regions of the observed lines. The time evolution of the ephemeral region in particular shows clearly the emergence of the bipolar structure through the photosphere and, eventually, into the chromosphere, confirming the scenario of opposite polarities in the photosphere and chromosphere.

The present paper gives an observational evidence of the evolution of an ER. The other studied structure is an 


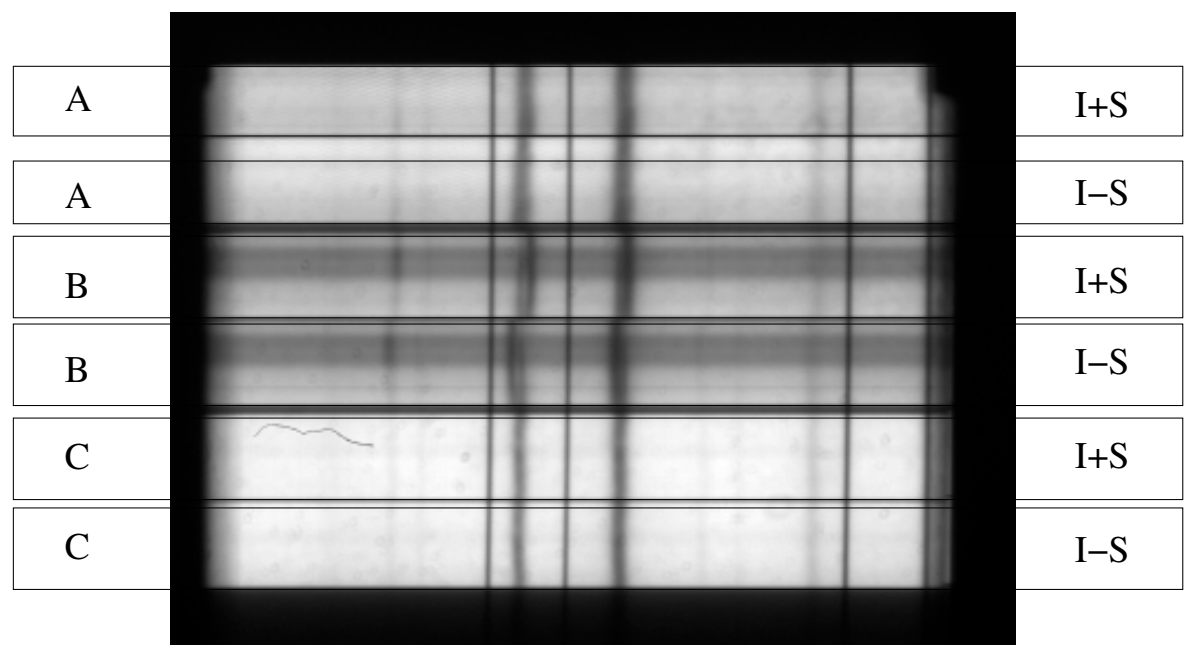

Fig. 1. An example of raw data from the THEMIS MTR observing mode. The capital letters correspond to the same observed area on the Sun with two orthogonal polarization states $I+S$ and $I-S$, where $S$ can be $+Q,+U,+V,-Q,-U$, or $-V$. The image shows the polarization state $S$ equal to $+V$; therefore, the pair of band shows $I+V$ and $I-V$.
MMF candidate. These structures were observed simultaneously in the photosphere using the Fe I 6301 and $6301 \AA$ lines and in the chromosphere using the Ca II IR triplet lines, thanks to the MTR mode of the THEMIS telescope.

The data of the MTR mode in THEMIS have long suffered from the lack of suitable standard tools for data reduction and analysis. This paper presents the first use of the new standard data reduction package for spectropolarimetric data of THEMIS, called SQUV (henceforth made available to THEMIS users); for this reason we dedicate some time to explaining the data reduction in Sect. 2.

We focus in Sect. 3 on the Stokes profiles of the ephemeral region and its inversion (for the photospheric lines only) and propose a scenario for the emergence of the bipolar structure that fits all the observed constraints. We shall discuss that scenario in the frame of current knowledge of ephemeral regions and their emergence in the final section.

\section{Observations and data reduction}

We observed the active region NOAA 10661 on 2004 August 17 (N07E21). This region showed two sunspots of the same positive polarity, resulting from the splitting of a single sunspot (NOAA 10652) one solar rotation before our observations. The trailing negative polarity was only visible as plages, but did not result in any sunspot. The observations were made with THEMIS (Rayrole \& Mein 1993) at the Observatorio del Teide in Tenerife. We used the MTR (multiwavelength spectropolarimetry) mode (López Ariste et al. 2000) in the setup called Semel's mask with a choice of four spectral regions observed simultaneously. Those regions were the triplet of Ca II IR lines and the region around the Fe I 6301 and $6302 \AA$ lines. The Semel's mask setup involves the use of a particular mask at the F1 focus of the telescope, before the polarimeter, which hides three segments of the solar image 17 arcsec wide so that the polarimeter places the image with orthogonal polarization in that region (see Fig. 1). With this setup, one can ensure the co-spatiality (Meunier \& López Ariste 2002) of both orthogonal-polarization images. The trade-off with this setup comes from the necessity of also scanning in the direction along the slit to fill the regions hidden by the mask. When using such a double scan, a rebuilding procedure is necessary to recover the real image over the Sun during data reduction.

The scanning step in the direction across the slit was 0.8 arcsec while the step in the direction along the slit was
16 arcsec. The spatial sampling of our cameras was of $0.45^{\prime \prime} / \mathrm{px}$, providing a theoretical spatial resolution slightly better than the slit (opened to 1 arcsec) and the median seeing during the observation (also 1 arcsec). The spectral dispersion was 21.9, 34.2 and $31.9 \mathrm{~m} \AA / \mathrm{px}$ for the 6302,8498 and $8542 \AA$ regions respectively.

In our setup we measure simultaneously over the camera detector two orthogonal polarizations and then modulate in time to complete all the polarization states, the sequence of modulation was in our case $I+Q, I-Q, I+U, I-V$ (referring always to the top beam). Figure 1 shows the raw data as it appears in our cameras.

The data were reduced by an online reduction package called Stokes QUick Viewer (hereafter SQUV). It is a graphical user interface developed in IDL that allows the reduction of THEMIS MTR data, in all its possible setups. Since it is intended to become the standard data reduction tool for THEMIS, we present here the main ideas behind it ${ }^{1}$.

SQUV uses the demodulation matrix technique (Collados 1999) to retrieve the Stokes paramaters from a given sequence of modulation of the polarization. In the case of a polarization sequence with a number of states less than four the code offers to just clean the image, i.e. to apply just the corrections from dark current and flat-fielding, hereafter DC and FF. In the more usual case where four or more polarization states have been measured, a demodulation matrix is calculated taking into account the observed wavelength, the THEMIS polarization analyser characteristics (refraction index and thickness of the waveplates) and the rotation state.

Written in equations, we denote the raw measurements $\widetilde{\mathbf{S}}$. These can be converted into the Stokes vector $\mathbf{S}$ through the use of a demodulation matrix $\mathbf{D}$, which is just the inverse of a matrix formed with the first raw of the Mueller matrix $\mathbf{M}$ for each one of the $k$ different states of the polarization sequence (Eqs. (1) and (2)).

$$
\underbrace{\left[\begin{array}{c}
I \\
Q \\
U \\
V
\end{array}\right]}_{\mathbf{S}}=\underbrace{\left[\begin{array}{llll}
M_{11,1} & M_{12,1} & M_{11,1} & M_{11,1} \\
M_{11,2} & M_{12,2} & M_{11,2} & M_{11,2} \\
M_{11,3} & M_{12,3} & M_{13,3} & M_{13,3} \\
M_{11,4} & M_{12,4} & M_{11,4} & M_{14,4}
\end{array}\right]^{-1}}_{\mathbf{D}} \underbrace{\left[\begin{array}{c}
\widetilde{S}_{1} \\
\widetilde{S}_{3} \\
\widetilde{S}_{3} \\
\widetilde{S}_{4}
\end{array}\right]}_{\widetilde{\mathbf{S}}}
$$

$\mathbf{S}=\mathbf{D S}$

${ }^{1}$ SQUV can be downloaded at the THEMIS website www. themis.iac.es 
The demodulation matrix $\mathbf{D}$ is retrieved using a single value decomposition for the matrix inversion once the Mueller matrix has been computed for a particular instrumental setup. This technique automatically finds the best choice for $\mathbf{D}$ in terms of noise and crosstalk (since for more than four states of polarization in a sequence, the inversion is not unique).

When using this demodulation method, it is not strictly necessary to use flat-field thechniques for Stokes $Q, U$ and $V$, since the demodulation implicitly applies them by comparing different polarization states measured over the same pixel regions. Nevertheless, SQUV does need the flat-field images to compute the curvature of the spectra and the position of the mask bands. The curvature is computed as the position of the minimum of a given spectral line interpolated with cubic splines (if SQUV just cleaned the data it would make use of the flat-field image in the traditional manner).

After demodulating, the Stokes images are compressed and deslanted with the positions and curvature computed from the flat-field. At this point two orthogonally polarized images per Stokes parameter are still available, one per each beam of the Semel's mask. A spatial correlation of each pair of images allows to subtract them, increasing the $\mathrm{S} / \mathrm{N}$ ratio while rejecting some spurious signals due to changes in time through the modulation cycle. For Stokes $I$ images, SQUV obviously adds, rather than subtracts, the bands. SQUV eventually writes the Stokes parameters in a standard FITS file.

An example of the data after demodulation and geometry corrections is given in Fig. 2, which shows the Stokes parameters at one raster position for all four spectral domains observed The horizontal axis corresponds to the spectral and the vertical axis to the spatial direction. Only one out of three bands available with the Semel's mask is shown in the figure for the sake of clarity. SQUV provides this kind of data-cube for each step of the scan and for every wavelength. Polarization fringes can be detected in some of the Stokes parameters at certain wavelengths. These fringes in polarization are due to the interferences at the waveplates of the ordinary and/or the extraordinary beams, as described by Semel (2003). SQUV does not make any attempt to defringe the data on the argument that we lack any quantitative information at this point on the parameters of those fringes; therefore, any defringing process would imply modelling and, necessarily, the ad hoc introduction of further information into the data.

For the other THEMIS MTR observing modes, the socalled $2 \times 2$ (orthogonal-polarization images 2 arcsec wide on two different cameras) and $2 \times 1$ (orthogonal-polarization images 1 arcsec wide in the same camera), the reduction procedure is similar. SQUV needs the flat-field of both images for calculating the curvature of the spectra and other geometrical parameters. The demodulation is done analogously to the Semel's mask case.

In this study we mostly constrain the analysis to the Stokes profile shapes. This is so because at this point we lack tools to infer magnetic field vectors for the chromospheric lines. We have nevertheless made use of the THEMIS-provided inversion code for the Fe I lines to retrieve some information on the photospheric vector magnetic field (López Ariste et al. 2006). The inversion tool, a fast PCA-based inversion code (Rees et al. 2000; López Ariste \& Casini 2002), provides a good approximation to the photospheric magnetic field in a standard Milne-Eddington atmosphere. It returns other than $\mathbf{B}$, its filling factor and the lineof-sight velocity $v_{\text {los }}$.

\section{Reversed-polarity profiles}

Among the many instances of chromospheric reversals in the polarity of Stokes $V$ found in our data, we have selected two structures.

The first of those structures is located close to the penumbral edge. It was observed at 14:51 UT on 2004 August 17. Figure 3 shows the intensity images (top) and the magnetograms (bottom) of the AR at this time made in the Ca II 849.8 and $854.2 \mathrm{~nm}$ lines and in the FeI doublet region at $630 \mathrm{~nm}$; a circle marks the position of the structure. At the photospheric level (see the two rightmost maps, top and bottom rows, Fig. 3), it shows an opposite polarity to that of the parent sunspot. The first of those structures is located close to the penumbral edge. It was observed at 14:51 UT on 2004 August 17. Figure 3 shows the intensity images (top) and the magnetograms (bottom) of the AR at this time made in the Ca II 849.8 and $854.2 \mathrm{~nm}$ lines and in the FeI doublet region at $630 \mathrm{~nm}$; a circle marks the position of the structure. At the photospheric level (see the two rightmost maps, top and bottom rows, Fig. 3), it shows an opposite polarity to that of the parent sunspot.

It should be said that almost all the points in the photospheric magnetogram found in similar conditions (opposite polarity to the sunspot parent and nearby to the penumbral edge) show the chromospheric reversal in polarity. That is, the chromosphere shows the same polarity as the parent sunspot. These structures are most probably moving magnetic features (Sheeley 1969; Harvey \& Harvey 1973)2 .

Figure 5 shows the intensity profiles ${ }^{3}$ of this MMF, as well as the $V / I_{\mathrm{c}}$ profiles. The reversal of the $V / I_{\mathrm{c}}$ profile at $8498 \AA$ is close to noise level but still clear. More evident is the reversal in the Ca II line at $8542 \AA$. The absence of any emission in the intensity profiles eliminates the possibility that the polarity reversal is due to an emission with no change of the magnetic polarity (Briand \& Vecchio 2003; Sánchez Almeida 1997). Such an eventuality could only be discarded after inspection of the full Stokes $I$ and $V$ profiles. It is obvious that absorption and emission lines will produce Stokes $V$ profiles opposite in sign for the same magnetic polarity. However, more often in the chromosphere, it is not the full line that goes into emission, but rather small cores occupying a small fraction of the resolution element and often Doppler-shifted respect to the background absorption line. In such cases the Stokes profiles are a linear combination of antisymmetric profiles changed in sign whether corresponding to emission, Doppler-shifted and scaled by the correspondent filling factor of the emission feature (Socas-Navarro et al. 2000). Examples of such behaviour in our data are found in the plage region in the lower left corner of the chromospheric intensity maps in Fig. 3. The sampling wavelength chosen to build those maps coincided with one of the spectral emission features in the chromospheric lines when in plages that consequently appear brighter than other parts. The corresponding Stokes $V$ profiles show the anomalous shape described above. Any attempt to perform classical filter magnetometry on those regions will fail or, even worse, will produce spurious signatures. In our chromospheric maps we paid attention to those problems and represented a clear magnetogram polarity just in the cases in which

2 The PCA inversion code returns the next values for this point: $B=$ $325 \pm 100 \mathrm{G}, \Theta_{B}=81 \pm 30$ degrees, $\Phi_{B}=45 \pm 25$ degrees, where $B$ is the strength of magnetic field in gauss, $\Theta_{B}$ is the inclination with regard to the vertical and $\Phi_{B}$ is the azimuth with 0 points towards the top the image.

A quiet sun profile has been overplotted as reference in all $I / I_{\mathrm{c}}$ plots. 


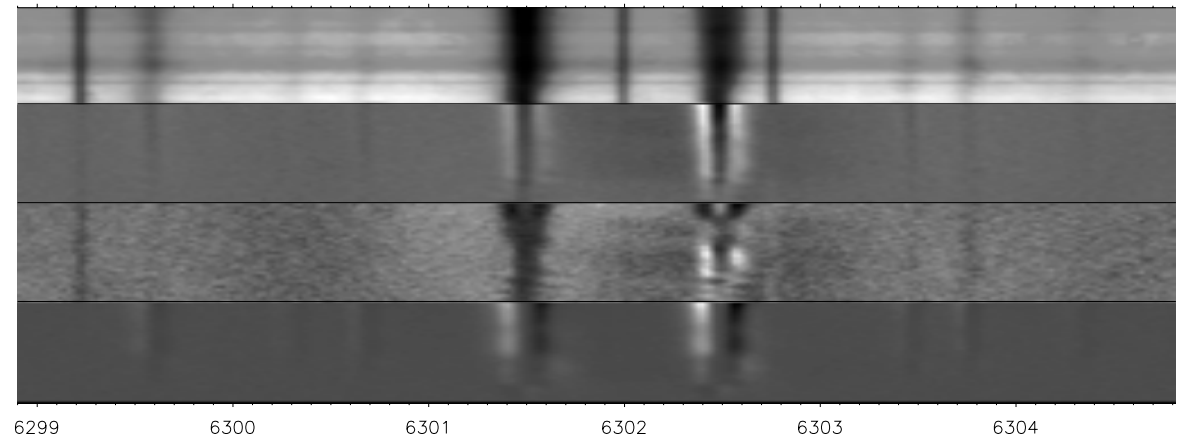

6299

6300

6301

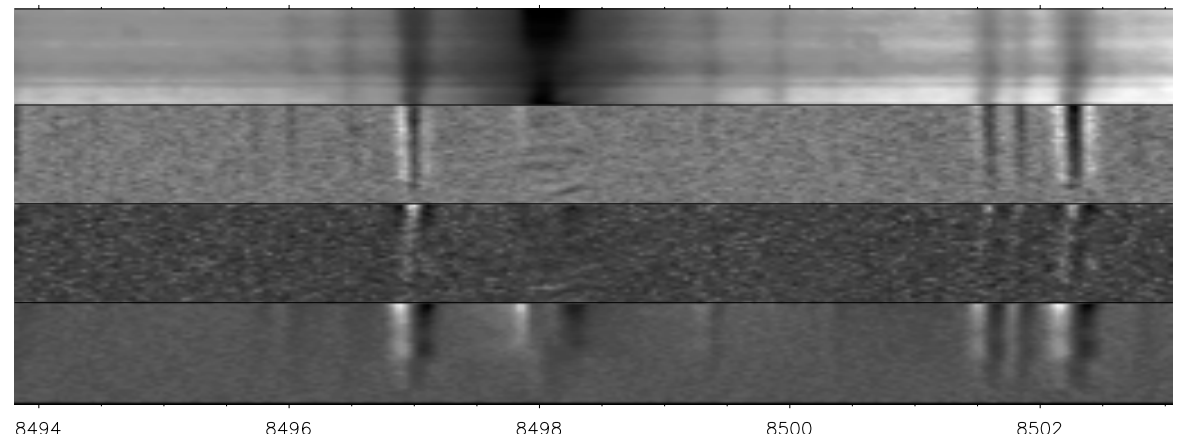

8494

8496

8498

8500

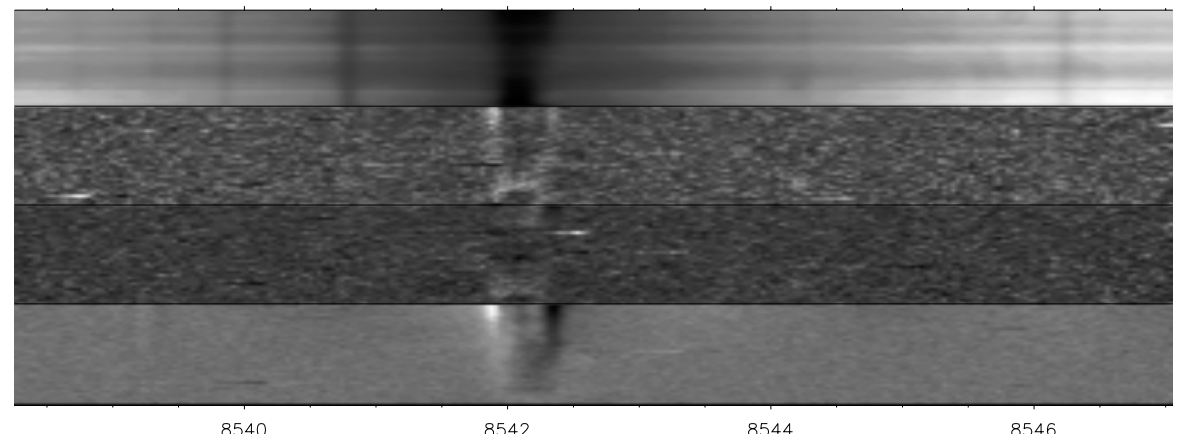

8540

8542

8544

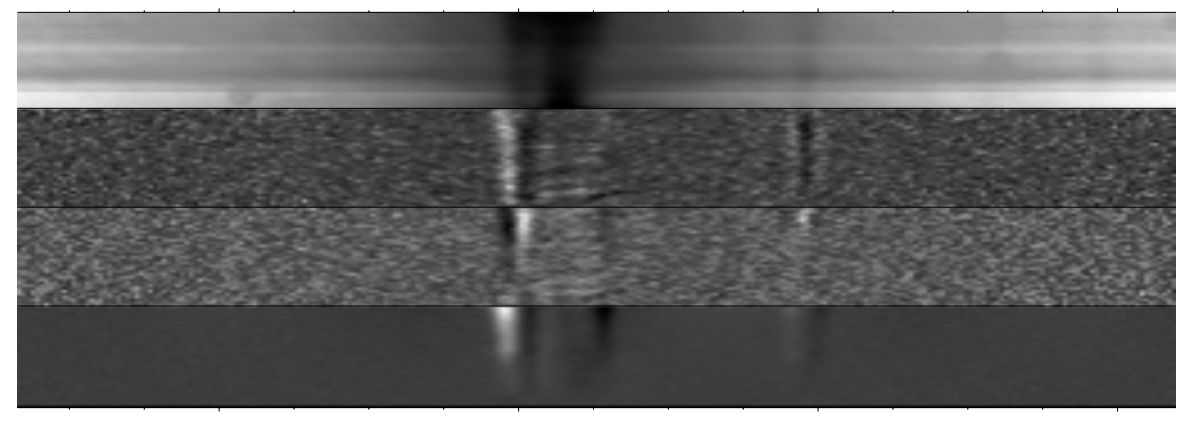

8660
8664
Fig. 2. The Stokes parameters, from top to bottom $I / I_{\mathrm{c}}, Q / I_{\mathrm{c}}, U / I_{\mathrm{c}}$ and $V / I_{\mathrm{c}}$, returned by SQUV for the simultaneous observation at 6301, 6302, 8498, 8542 and $8662 \AA$. an antisymmetric profile could be found. Whenever anomalous Stokes $V$ profiles were present the magnetogram map shows a chaotic mixture of polarities that should not be overinterpreted as polarity changes over the Sun, but rather as the generalized presence of emission features over the line. This is the great danger of chromospheric magnetograms and this is the reason for our emphasis on the actual full Stokes profiles as the only manner to ascertain the physical scenarios in place.

Now that anomalous profiles have been discarded, let us focus again on the MMF. At the photosphere, its polarity is opposite to that of the corresponding sunspot parent. However, the polarity of this point at the chromosphere along the line of sight is the same as for the sunspot: we are observing the canopy. The topology of the magnetic structure related to the MMF does not therefore reach the chromosphere in this case. Following the classification given by Shine \& Title (2001) the observed MMF is of the type III. It is just a photospheric perturbation. In Fig. 4 we suggest the possible topology of the MMF and sunspot canopy structures. We have computed the derivative of the $8542 \AA$ intensity profile and have used the weak-field approximation to fit the $V$ profile calculated as for the observed one. The $B_{\text {los }}$ obtained is $120 \mathrm{Mx} / \mathrm{cm}^{2}$. 

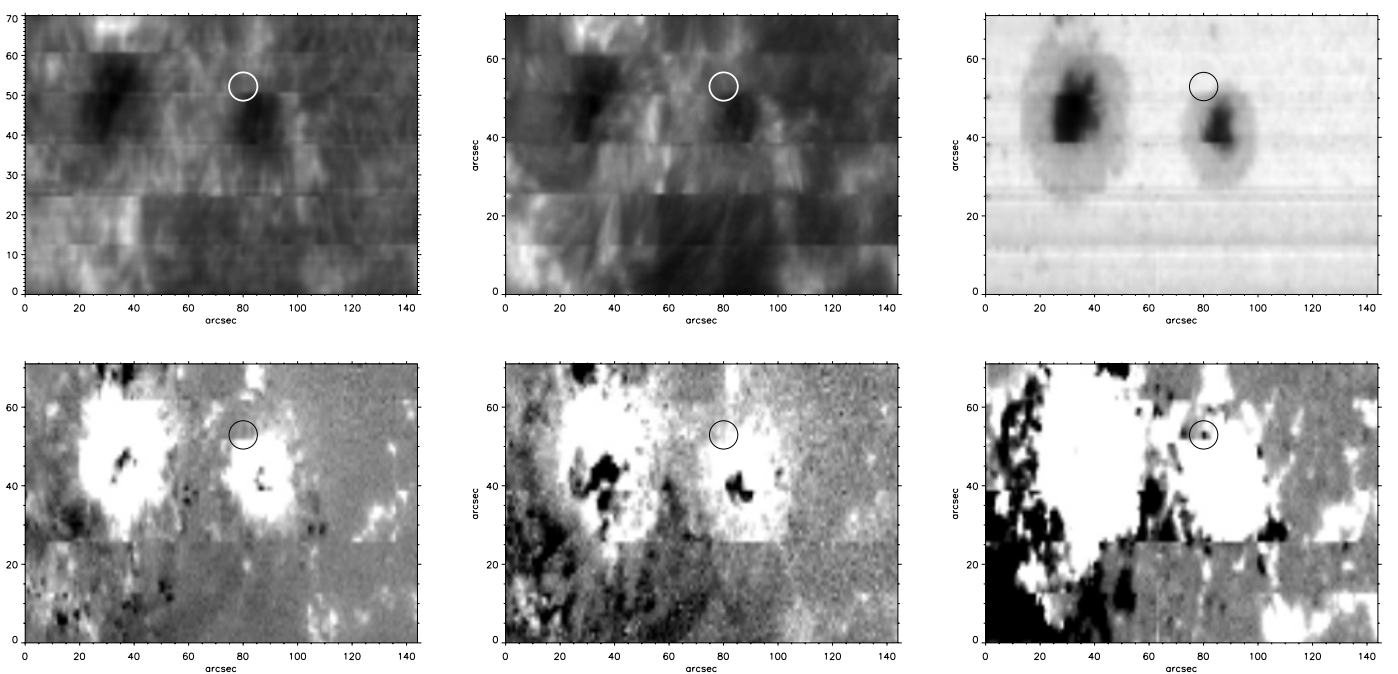

Fig. 3. Reconstructed intensity images (top) from the core of the Ca II $8498 \AA$ (left) and $8542 \AA$ lines (centre) and at continuum of Fe I 6301 \& $6302 \AA$ lines and magnetograms (bottom) from Stokes $V$ profiles for the same spectral ranges of NOAA AR 1662 on 2004 August 17, $14 \mathrm{~h} 51$ min. The circle marks the location of a candidate of MMF (opposite polarity with regard to parent sunspots). The photospheric profiles of this MMF show a reversed polarity with respect to the chromospheric ones.

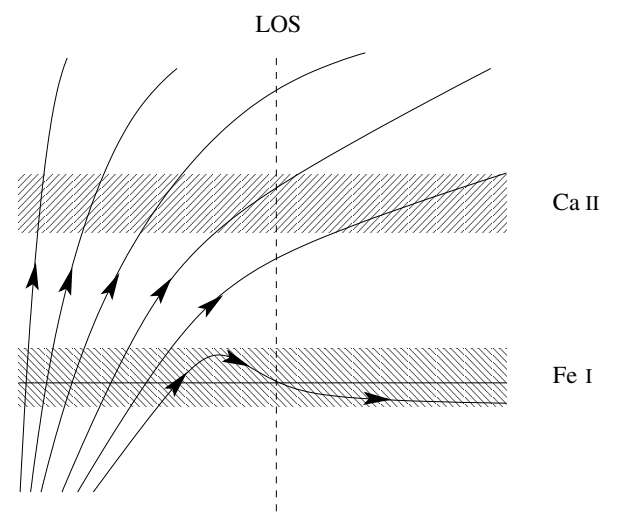

Fig. 4. A schematic view of a possible configuration for the observed MMF taking into account current knowledge of sunspot, canopy and MMF. The more vertical lines represent the umbral outer magnetic field lines. Next to these lines, the canopy lines spread out around the sunspot and reach the chromosphere (the Ca II layer). In the photosphere, the MMF is a small perturbation of the magnetic field line. The arrows show the sense of the magnetic field lines. The vertical dashed line is the line of sight. The phostosphere is represented by a horizontal line inside the Fe I layer. Sizes and distances are not to scale.

The second selected structure corresponds to the evolution in time of the positive polarity of an ephemeral region. It was first observed on 2004 August 17 at 17:23 UT. Figure 6 shows the ER marked by a circle in the intensity images and magnetograms. The corresponding profiles can be seen in Fig. 7. The reversal in polarity is clear. It coincides in time with a ratio of Stokes $V$ amplitudes in the photospheric Fe I lines which is very near to the ratio of their respective effective Landé factors $(0.65)$, indicating that they are in the weak-field regime. This is confirmed by the inversion of these lines that results in field strengths of $500 \mathrm{G}$.

The polarity reversal in the Ca II 8498 and $8542 \AA$ profiles is, as in the example of the MMF above, of a magnetic nature: while we note that the $I / I_{\mathrm{c}}$ profiles of Ca II show emission in the red wing of the lines, it is clear from its position in wavelength that it is not related to the reversal of the Stokes $V$ profile, clearly blue-shifted with respect to the emission.
This conclusion is strengthened by the evolution observed 32 min later. Figure 8 shows the second scan of the region. The polarity reversal has evolved into an anomalous profile (see Fig. 9), similar to those found in a polarity inversion line, where signals of both polarities are mixed in the same resolution element. Simultaneously, the photospheric field has increased in strength to the kilogauss level (the ratio between the Stokes $V$ profiles of Fe I 6301 and $6302 \AA$ can be seen to be roughly 1, in agreement with the inversion results) and it has become more vertical (from $53 \pm 18$ to $23 \pm 32$ degrees with respect to the vertical).

A zoom of the ER is shown in Fig. 10. We estimate that the white photospheric polarity of the ER has travelled $2600 \mathrm{~km}$ away from the negative polarity at an avarage speed of $1.4 \mathrm{~km} \mathrm{~s}^{-1}$. The area of both polarities of the ER has roughly decreased by a factor $3 / 4$.

Our interpretation of this evolution is sketched in Fig. 11. In the first scan we find an emerging ephemeral region (in the form of an $\Omega$-loop), which can be seen in the photosphere, with fields which are mostly horizontal. The chromosphere has not yet felt the upcoming ephemeral region and shows the predominant negative polarity of the region and a magnetic flux that can be estimated at $75 \mathrm{Mx} / \mathrm{cm}^{2}$ (from a weak-field approximation to the amplitude of Stokes $V$ of the Ca $8542 \AA$ line). $32 \mathrm{~min}$ later the magnetic field has become increasingly vertical and strong and has emerged at the chromosphere, where it can now be seen mixed with the predominantly negative polarity as seen by the Ca II lines. The picture is confirmed by the ratio of areas in the two times being 3/4, in good agreement with the ratio of the inclinations of the magnetic fieild $(\cos 53 / \cos 23 \approx 0.65)$. This points once more to the picture of an emerging $\Omega$-loop with a constant diameter of $2300 \mathrm{~km}$.

\section{Discussion}

The observed chromospheric reversals can be unambiguously attributed in the selected cases to true magnetic reversal from the photosphere to the chromosphere. This identification has allowed the sketch of the emergence of this particular ephemeral 

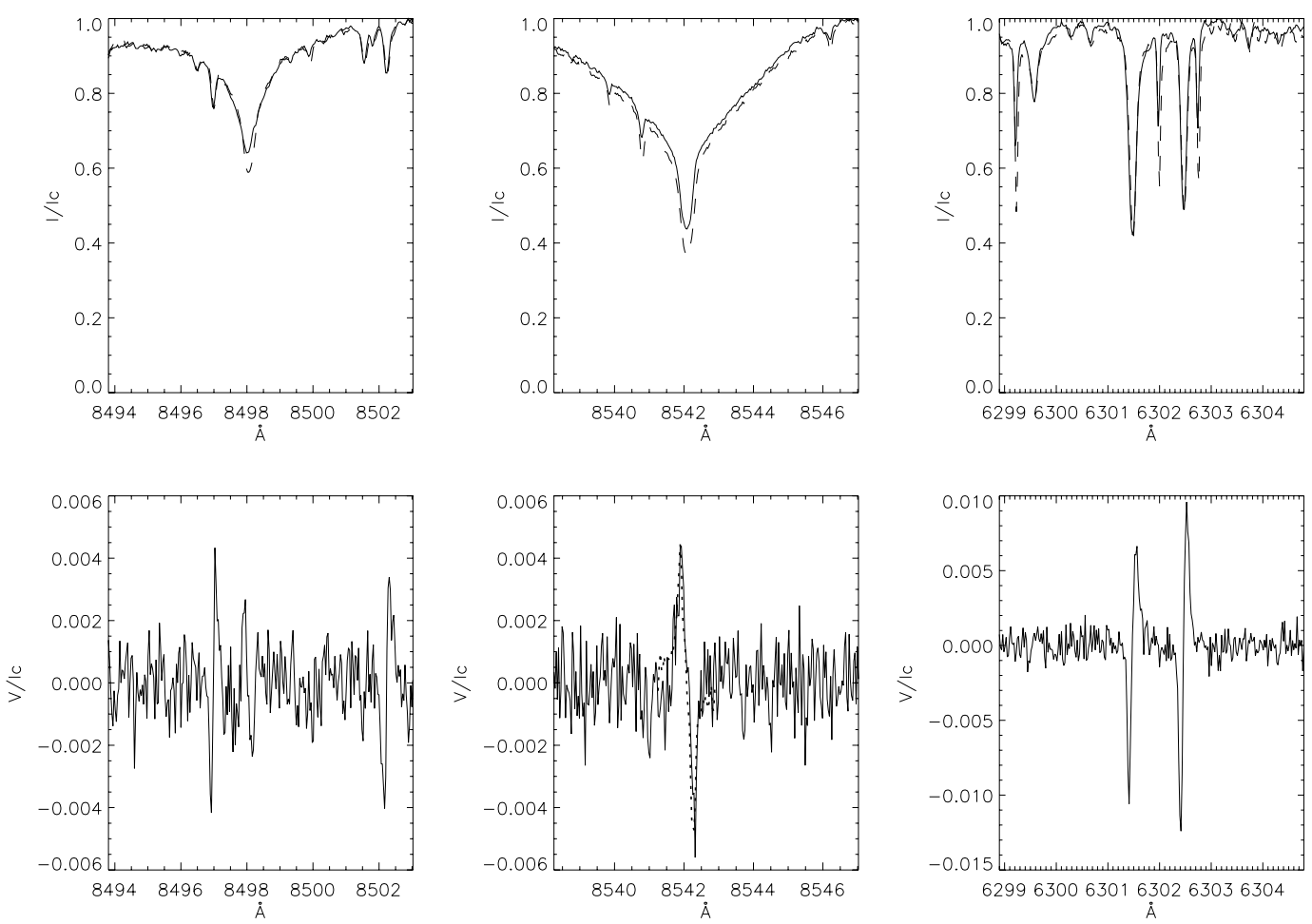

Fig. 5. Profiles of $I / I_{\mathrm{c}}(t o p)$ and $V / I_{\mathrm{c}}$ (bottom) of the MMF. A profile of quiet sun is overplotted for reference (dashed line). The Ca intensity profiles present no emission signatures but the $V$ profile at $8542 \AA$ is clearly reversed with respect to the $V$ profile of the photospheric lines in the $8498 \AA$ region or of the $6302 \AA$ one. The Ca line at $8498 \AA$ also shows the reversal but close to noise level. For $8542 \AA$ the $V$ profile calculated from the weak magnetic field approximation (making use of the derivate of intensity profile) has been overplotted (dot line): $B_{\text {los }}$ is roughly $120 \mathrm{Mx} / \mathrm{cm}^{2}$.
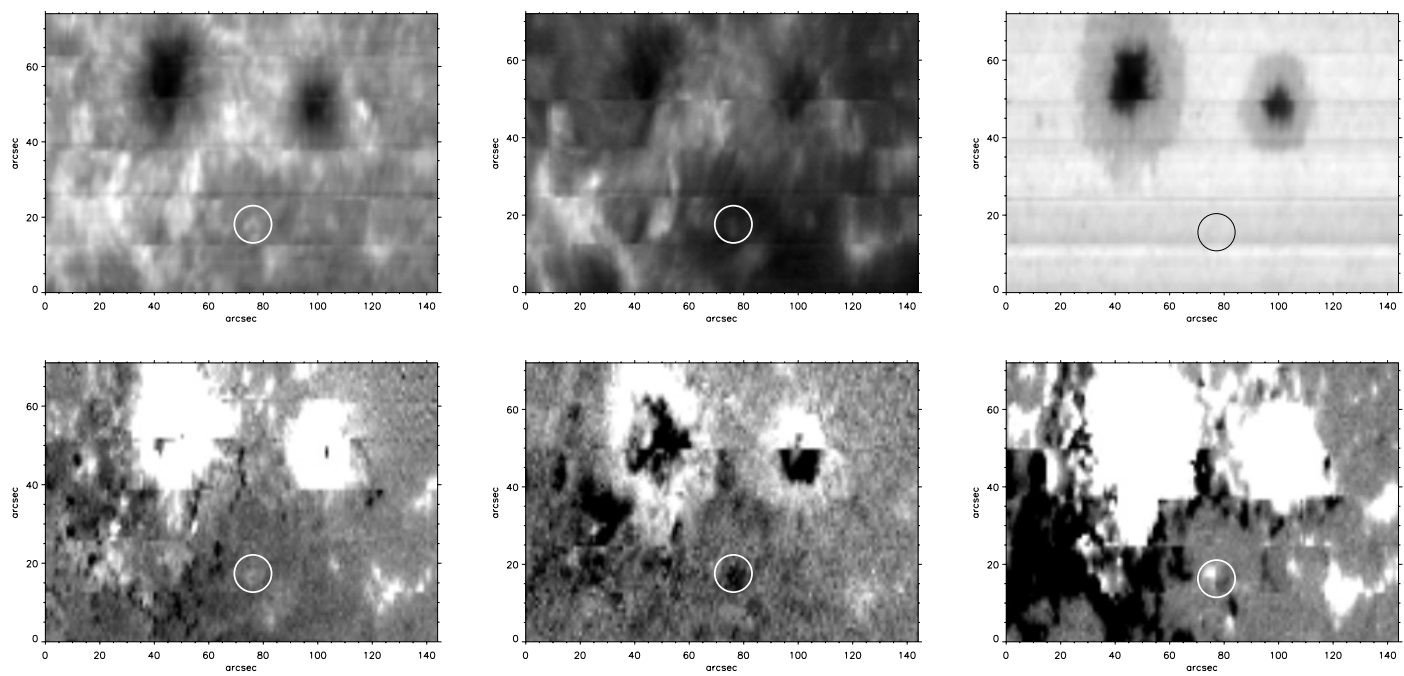

Fig. 6. Reconstructed intensity images and magnetograms (made as in Fig. 3) of NOAA AR 1662 on 2004 august 17, 17h23min for Ca II 8498 (l) \& $8542 \AA$ (c) and Fe I $6301 \& 6302 \AA$ (r). The circles show the location of an ephemeral region whose (photospheric) white polarity is studied.

region through the photosphere into the chromosphere, roughly $1000 \mathrm{~km}$ above.

We first recorded the emerging ephemeral region when the two polarities were 5 arcsec apart $(3.5 \mathrm{Mm})$. Although this is a significant distance, and they probably first emerged in the photosphere much nearer (Martínez Pillet 2002), it is also clear that the region is barely out of the formation region of the Fe lines. We conclude this from the strong inclination of the field in the positive polarity and its weak strength. The negative polarity (of which we have not shown profiles here) shows even noisier profiles. In this process the region is obviously very asymmetric in its polarities.

The two polarities separated by an average speed of $1.4 \mathrm{~km} \mathrm{~s}^{-1}$ in the subsequent $32 \mathrm{~min}$, while the field intensified by a factor of 3 and became more vertical in both polarities. This behaviour, now confirmed by the inversions, has been described before (Schrijver et al. 1997) and is a natural consequence of the rising of the $\Omega$-loop and the buoyancy of the stronger fields. Furthermore, the area of each polarity shrinks by $3 / 4$ in that time, otherwise corresponding to the rising of a constant-diameter flux 

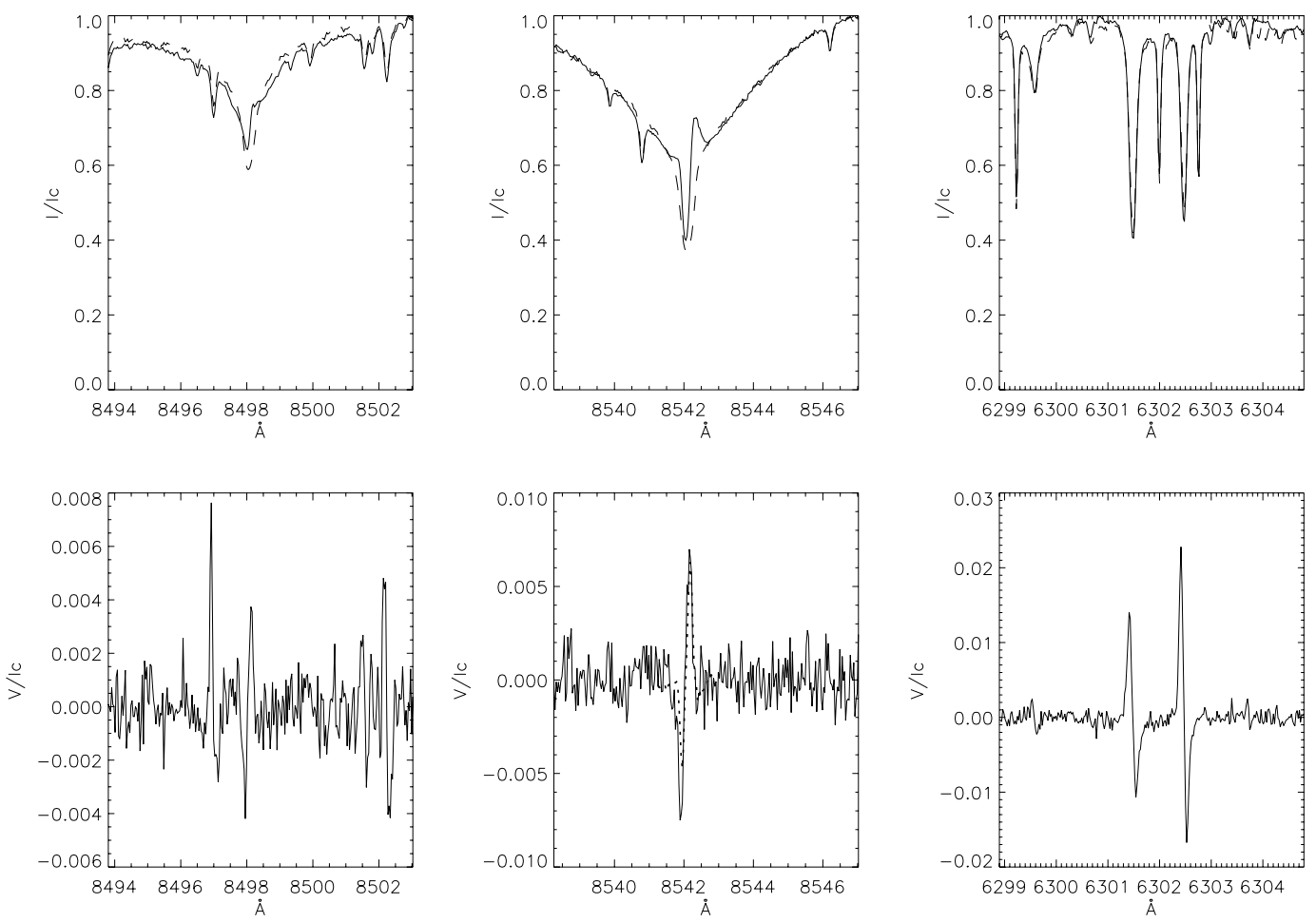

Fig. 7. Stokes $I$ and $V$ profiles corresponding to the first observation of the ephemeral region white polarity. A profile of quiet Sun is overplotted for reference (dashed line). The intensity profiles (top) of Ca II IR lines show emission in the wings. The reversal on the Stokes $V$ profiles (bottom) of these lines with respect to the photospheric lines is clear, but not related to the emission. The amplitude ratio of $V / I_{\mathrm{c}}$ for the Fe I 6301 and $6302 \AA$ lines points to weak photospheric fields. In the chromosphere, a weak-field approximation measurement for $8542 \AA$ results in a $B_{\text {los }}$ of roughly $75 \mathrm{Mx} / \mathrm{cm}^{2}$ (the fit to the $V$ profile computed in this approximation is overplotted with a dotted line).
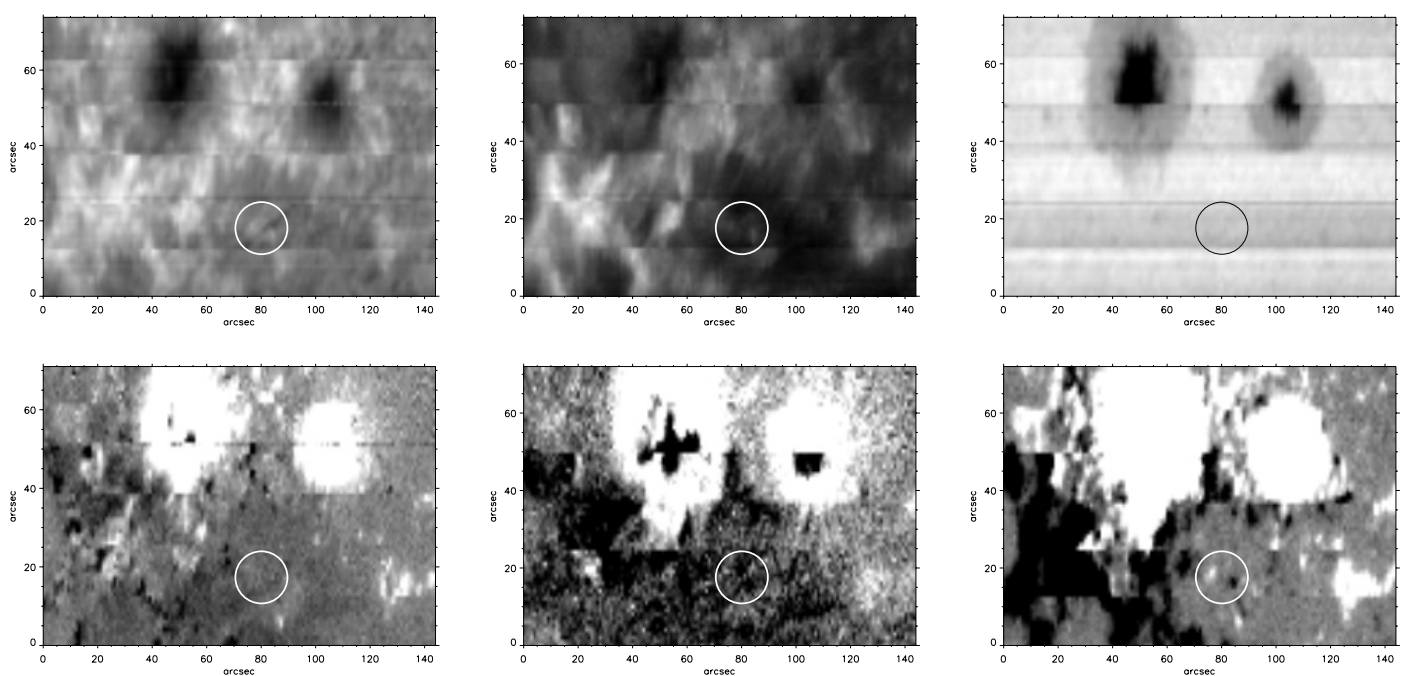

Fig. 8. Reconstructed intensity images and magnetograms (made as in Figs. 3 and 6) of NOAA AR 1662 on 2004 August 17, 17h51min for Ca II $8498 \AA$ (l) \& $8542 \AA$ (c) and Fe I $6301 \& 6302 \AA$ (r). The white (photospheric) polarity pole of the ephemeral region has increased its magnetic strength and separated from the other pole.

tube with the inclination varying from 53 to 23 degrees. But we also see that in those 32 min the emergent field has barely been able to reach the formation region of the $\mathrm{Ca}$ II lines in the chromosphere since no signs of it are seen in the first observation and we observed mixed polarities in the second observation. It is clear that the $\Omega$-loop is not rising into the corona at the same rate as the two polarities travelling apart in the photosphere. Either the thermodynamic conditions at the minimum of temperature are slowing down the perturbation in height or the coronal field (negative polarity) is preventing emergence. Without more sophisticated analysis of the chromospheric data, it is impossible to tell the two scenarios apart.

The chromospheric lines do show some emission in the line core in the first observation. We are mostly tempted to disentangle this emission from the emergence of the $\Omega$-loop. It is redshifted and therefore could tentatively be attributed to some down-going perturbation exciting the chromosphere sufficiently for the Ca II atoms to revert to emission. Whatever its origin, it has mostly disappeared by the time the $\Omega$-loop reaches chromospheric heights. At this point there is no visible 

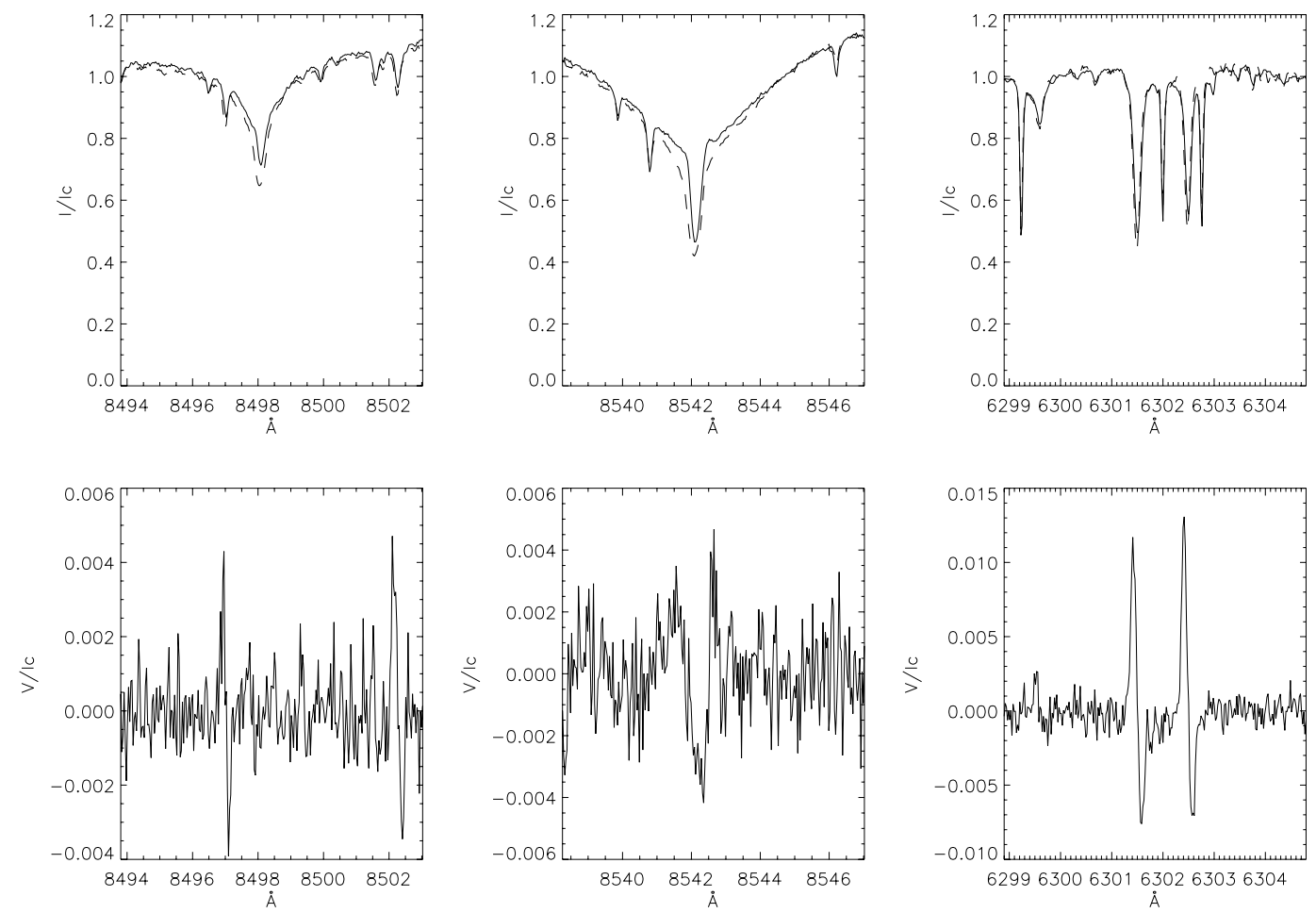

Fig. 9. Stokes $I$ and $V$ profiles corresponding to the second observation of the ephemeral region white polarity. A profile of quiet Sun is overplotted for reference (dashed line). The Stokes $V$ profiles at $8542 \AA$ present an anomalous shape characteristic of neutral lines. In the photosphere the ratio of $V$ amplitudes points to a stronger field.
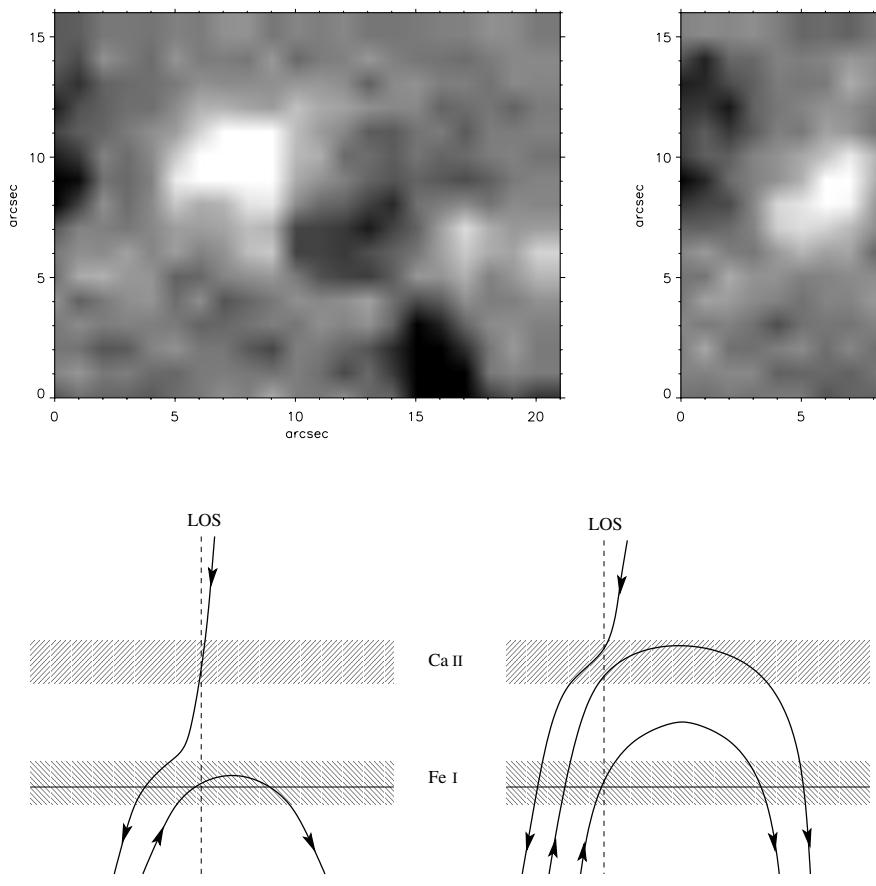

Fig. 11. Sketch of the emergence of the observed ephemeral region. Left: the top of the $\Omega$-loop is close to the photosphere (horizontal line in the dashed Fe I formation layer), while the chromosphere is embedded in the background magnetic field of the active region trailing polarity, with opposite polarity. Right: 32 min later, the top of the loop has reached the chromosphere and a cancellation process is observed in the $\mathrm{Ca}$ profiles, while in the photosphere the field has become stronger and more vertical. Sizes and distances are not to scale.

thermodynamic signature of any reconnection taking place (Archontis et al. 2005) and it is clear that the loop has not reached further heights at this time. In this particular case, therefore, the ephemeral region has not succeeded, at the time of observation, in reconnecting with the coronal field. Neither signature shows evidence of any reconnection at the photospheric level in the regions probed by the Fe lines.

While we have presented the example of the MMF mainly for illustrative purposes, it is important to stress that it would be valuable to explore as well its signatures at the chromospheric levels since they have been related at times with the $\mathrm{H} \alpha$ Ellerman bombs (Nindos \& Zirin 1998; Georgoulis et al. 2002). At the time of our observations the studied MMF had not reached the chromosphere and could not be linked to any chromospheric process.

Acknowledgements. Thanks are due to V. Martínez Pillet and B. Gelly for carefully reading this manuscript, and also to R. Manso Sainz for his comments and ideas on the profiles and schematic diagrams. Ideas given by V. Pillet Martínez and M. Collados on the reduction data and demodulation techniques were very useful. The authors are grateful to T. Mahoney for the linguistic and 
edition revision. We wish to thank the THEMIS staff for their work during the observations, with particular mention of the acting telescope operator and the support astronomer.

\section{References}

Archontis, V., Moreno-Insertis, F., Galsgaard, K., \& Hood, A. W. 2005, ApJ, 635, 1299

Briand, C., \& Vecchio, A. 2003, A\&A, 403, L33

Collados, M. 1999, Third Advances in Solar Physics Euroconference: Magnetic Fields and Oscillations, ASP Conf. Ser., 184, 3

Dodson, H. W. 1953, PASP, 65, 256

Georgoulis, M. K., Rust, D. M., Bernasconi, P. N., \& Schmieder, B. 2002, 575, 506

Hagenaar, H. J. 2001, ApJ, 555, 448

Harvey, K., \& Harvey, J. 1973, 28, 61

Harvey, K. L., \& Martin, S. F. 1973, Sol. Phys., 32, 389

López Ariste, A., \& Casini, R. 2002, ApJ, 575, 529

López Ariste, A., Rayrole, J., \& Semel, M. 2000, A\&AS, 142, 137
López Ariste, A., Aulanier, G., Schmieder, B., \& Sainz Dalda, A. 2006, A\&A, 456, 725

Martin, S. F. 1990, in IAU Symposium, ed. J. O. Stenflo, 129

Martínez Pillet, V. 2002, in Solar-Terrestrial Magnetic Activity and Space Environment, ed. H. Wang, \& R. Xu, 9

Meunier, N., \& López Ariste, A. 2002, THEMIS Workshop, ed. J. Arnaud, \& N. Meunier, 121

Nindos, A., \& Zirin, H. 1998, 182, Issue 2, 381

Rayrole, J., \& Mein, P. 1993, The Magnetic and Velocity Fields of Solar Active Regions, IAU Colloq., 141, ASP Conf. Ser., 46, 170

Rees, D. E., López Ariste, A., Thatcher, J., \& Semel, M. 2000, A\&A, 355, 759

Sánchez Almeida, J. 1997, A\&A, 324, 763

Schrijver, C. J., Title, A. M., van Ballegooijen, A. A., Hagenaar, H. J., \& Shine, R. A. 1997, ApJ, 487, 424

Semel, M. 2003, A\&A, 401,

Sheeley, N. R. 1969, Sol. Phys., 9, 347

Shine, R., \& Title, A. 2001, in Encyclopedia of Astronomy and Astrophysics, (London: Nature Publishing Group, and Bristol: Institute of Physics Publishing), 4, 3209

Socas-Navarro, H., Trujillo Bueno, J., \& Ruiz Cobo, B. 2000, ApJ, 544, 1141 